\title{
LA APLICACIÓN DE LOS “MODOS TECNOLÓGICOS” EN EL ANÁLISIS DE LAS INDUSTRIAS PALEOLÍTICAS: REFLEXIONES DESDE LA PERSPECTIVA EUROPEA
}

\author{
THE APPLICATION OF "TECHNOLOGICAL MODES" IN THE ANALYSIS OF \\ PALAEOLITHIC INDUSTRIES. REFLECTIONS FROM A EUROPEAN PERSPECTIVE
}

por

FERNANDO DIEZ MARTÍN*

EnSUMEN este artículo se presenta un análisis crítico sobre las aportaciones y limitaciones del esquema
de los Modos tecnológicos de Grahame Clark para el estudio del Paleolítico inferior y medio.

\begin{abstract}
A critical analysis is presented in this paper on the contributions and limits related to Grahame Clark's technological Modes model for the study of the Lower and Middle Palaeolithic. The need of its redefinition is pointed out and some new perspectives are proposed.
\end{abstract}

Palabras claves Paleolítico inferior y medio, olduvayense, achelense, musteriense, Modos tecnológicos 1,2 y 3 .

Key words L Lower and Middle Palaeolithic, Oldowan, Acheulean, Mousterian, Technological Modes 1,2 and 3.

\section{INTRODUCCIÓN}

El estudio del Paleolítico europeo ha estado fuertemente influido por una concepción evolucionista e historicista, que se ha visto particularmente reflejada en el análisis y la interpretación de las tecnologías más antiguas. Dada la fuerza ideológica y el protagonismo de las corrientes evolucionistas en la historia del pensamiento occidental (Adams 1998: 9-73) no podemos sorprendernos del papel que éstas han jugado en el estudio de la Prehistoria y el modo en el que las caracterizaciones de los objetos arqueológicos se han guiado por la relación simple/complejo (Torrence 1989). La perspectiva gradualista, plenamente vigente en los albores decimonónicos de nuestra disciplina, sigue manifestándose aún, menos nítidamente, en las preferencias de algunos investigadores.

* Área de Prehistoria. Universidad del País Vasco. C/Francisco Tomás y Valiente, s/n. Apartado 21 11.01006 Vitoria-Gasteiz. fdimar@lycos.com 
El caso de la línea historicista, no necesariamente ligada a la anterior, nos interesa más en esta reflexión. Su papel ha sido consustancial al origen y desarrollo de la ciencia prehistórica en Europa, hasta el punto que se ha convertido en su rasgo distintivo. Como bien es sabido, mientras que la arqueología norteamericana ha estado ligada desde sus comienzos a los intereses y planteamientos de la antropología (gracias a una prehistoria viva, en la figura del nativo americano, que difícilmente podía distinguirse de la etnología), la europea se ha relacionado, por tradición, con la historia y su discurso. Aquí, el Otro que hace las veces de nativo americano (Adams 1998: 6) se hundía en la noche de un pasado histórico efectivo. Esta concepción puede observarse de manera prominente en nuestra Prehistoria más antigua y en el modo en que se ha ido construyendo a lo largo de los años. La tradición paleolitista europea ha tendido preferentemente a historiar los vestigios por antonomasia de aquel pasado, la industria lítica. Ello se consiguió llevando al extremo la práctica de la agrupación, poniendo en relación yacimientos que compartían determinados rasgos o particularismos tipológicos. El uso de los yacimientos de referencia, o epónimos, para designar dichas asociaciones, generó un complejo entramado de relaciones y de listados toponímicos interminables. (fig. 1)

La figura 1 reproduce un modelo que puede servirnos como ejemplo ilustrativo de tal situación. Ciertamente, no se trata solo, aunque también sea así, de una procedimiento analítico que puede llegar a frustrar y agobiar al más entusiasta, perdido en una maraña de rasgos distintivos, de relaciones sincrónicas o influencias diacrónicas entre tecno-complejos, grupos, facies y subfacies que parece no acabar nunca (clactoniense, tayaciense, taubachiense, achelense, micoquiense, musteriense, charentiense, vasconiense, pontiniano, szeletiense...), sino que posibilita un comentario más amplio.

Esta perspectiva conlleva primordialmente un modelo de interpretación del registro arqueológico que confía en convertir los rasgos objetivos en entidades de carácter histórico. No en vano, cada recuadro de la figura 1 representa algo que ha sido identificado a menudo con nombres tan significativos y rotundos como cultura, tradición o civilización, reflejo de grupos humanos con entidades históricas, culturales y sociales cuya independencia y relación podría ser observada a partir de los datos arqueológicos ${ }^{1}$. Obviamente, éstos - también los vinculados a nuestro pasado paleolítico- evidencian en diversa medida interacciones sociales de algún tipo o pertenecen a entidades culturales que el investigador intenta poner de manifiesto. Un buen ejemplo de ello es el debate que, durante casi treinta años (Gowlett 1996: 155), se está llevando a cabo sobre el significado de los primeros yacimientos arqueológicos y la posibilidad de que en ellos se compartieran los recursos y, por tanto, se interactuará socialmente (una completa revisión de este tema puede verse en Kuhn y Sarther 2000).

En este caso nos referimos a la Cultura y Sociedad con mayúsculas, a la asunción de que los tipos y las agrupaciones líticas que éstos definen tienen valor como indicadores sociales de las relaciones espaciotemporales existentes entre grupos humanos (Shanks y Tilley 1987: 81). Collins (1969: 277), por ejemplo, considera que las tradiciones y los estadios paleolíticos deben ser interpretados análogamente a lo que Childe entendía por cultura cuando se refería a los primeros grupos agricultores: llamaremos grupo culturalo cultura a ese conjunto de rasgos regularmente asociados. Asumimos que tal complejo es la expresión material de lo que hoy sería un grupo humano específico (Childe 1929: v-vi). Esta perspectiva no solamente se observa en publicaciones que vieron la luz en momentos más acordes con esa nomenclatura, como pudiera ser el entonces fundamental y significativo trabajo de Breuil (1936), sino con posterioridad a la propuesta de renovación taxonómica presentada ya en los años sesenta en Bishop y Clark (1967). Así, por ejemplo, el achelense y sus industrias contemporáneas sin bifaces (clactoniense o tayaciense) se han visto como respuestas arqueológicas propias de grupos humanos culturalmente distintos (Tieu 1991: 93-102). El problema y la duda, en estos casos, reside en la medida en que la información social que podemos arrancar de los aspectos tecnológicos y económicos del registro puede revelar la existencia de estructuras culturales de tal magnitud.

1. Un ejemplo paradigmático de la perspectiva "tradición-cultura", como él mismo la define, lo constituye el análisis de Collins sobre el Paleolítico europeo (1969). 


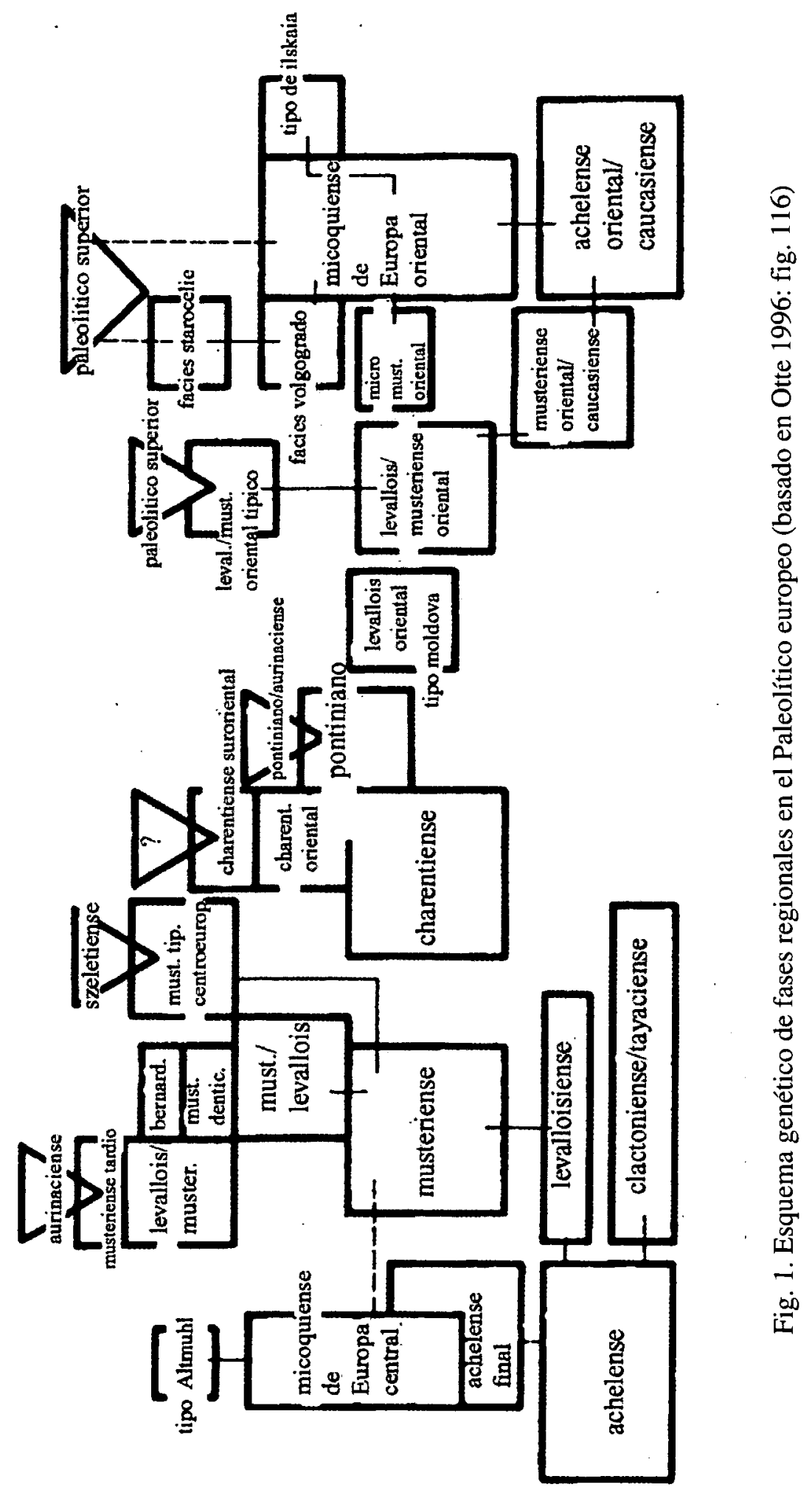


En los últimos años determinadas corrientes interpretativas, en absoluto nuevas, se han asentado con firmeza y los investigadores son más conscientes del complejo mundo de la variabilidad tecnológica y de los múltiples factores que pueden determinar las formas finales de los objetos, principalmente de naturaleza funcional o ambiental (Toth 1982: 3-4, Santonja 1996: 11). Estas perspectivas no pueden desligarse de la tendencia a aligerar los análisis líticos de una excesiva carga tipologista y del uso de nuevos esquemas de clasificación más preocupados por los procesos que por los objetos en sí. Como dice Gowlett (1996: 135): La tipología estática y clasificadora se ha acabado. Por el contrario, predomina una perspectiva más dinámica de acercamiento a la tecnología como práctica social. La popularidad masiva que ha alcanzado en los últimos años el concepto de las cadenas operativas y la profusión de su uso son fiel reflejo de ello (véase por ejemplo, Boëda et al. 1990, Panera y Rubio 1999).

Si los métodos de análisis de la industria se han visto transformados en diversa medida, es obvio que los modelos taxonómicos de referencia lo habrán hecho de forma similar. Ante una situación metodológica y teórica en la que predomina la diversidad, podríamos simplificar las opciones del siguiente modo: 1. aquellas en las que el esquema historicista sigue estando substancialmente presente, mostrando el poder atávico de su discurso, aunque con un lenguaje que recurre a menudo a terminologías más suaves, tales como facies o aspecto (Otte 1996); 2. las que situaríamos en un punto intermedio, manteniéndose fieles a la koiné terminológica clásica y a su valor universal, pero simplificándola y aligerando el uso de las facies regionales; 3 . las que han querido apartarse radicalmente del escenario histórico-cultural de la tradición europea y proponer nuevos marcos de comprensión de la realidad paleolítica.

\section{EL ESQUEMA DE LOS MODOS TECNOLÓGICOS DE GRAHAME CLARK COMO NUEVO HORIZONTE INTERPRETATIVO}

La actualidad y diversidad metodológica de la investigación paleolítica europea debe comprenderse, como hemos visto, en el marco de su particular andadura. En lo referente al universo conceptual de la evolución de las industrias líticas, y a su adscripción terminológica, la mayor parte de investigadores están optando por la segunda de las opciones propuestas anteriormente. Éstos aceptan el valor del lenguaje tradicional como medio universal de comunicación y dan por bueno el uso de conceptos estructurales o "complejos industriales" tales como el olduvayense o el achelense, mientras que las facies secundarias son apartadas, al verse como meros reflejos de la compleja variabilidad que caracteriza a las tecnologías paleolíticas.

Otros autores han sopesado más el valor de las palabras y de su carga histórico-cultural. Por ello han considerado que la mejor forma de desmarcarse completamente de aquella "agobiante" perspectiva historicista consistía en el cambio de las etiquetas y el abandono de la nomenclatura tradicional. No es de extrañar, por tanto, que estos investigadores optaran por revitalizar precisamente el esquema de los modos tecnológicos propuesto por Grahame Clark (1977), arqueólogo británico asociado a la escuela ecológica de los años $50^{2}$. Considerar que esta tendencia representa simplemente una posición simbólica de ruptura con la "tradición" sería simplificar la realidad en demasía, puesto que los relativamente escasos investigadores europeos que han optado por utilizar el esquema de los modos tecnológicos han tomado posiciones significativamente alternativas y rupturistas con los esquemas tradicionales.

Entre ellos hay que hacer mención de Foley y Lahr(1997), que partiendo de ideas expresadas anteriormente (Foley 1987), defienden que la tecnología paleolítica puede estudiarse desde una perspectiva filogenética,

2. Junto a la ecología cultural de los norteamericanos Julian Steward o Gordon Willey, la escuela ecológica de mediados del siglo XX comparte el interés por los aspectos medioambientales del registro arqueológico (fauna, flora, territorio y paisaje) frente a la tradicional perspectiva histórico-cultural y su interés por las tipologías (Renfrew y Bahn 1991: 33). 
análoga a los procedimientos cladísticos usados por la antropología física. Esta concepción pretende desligar los análisis tecnológicos de la influencia historicista y alinearlos con modelos propios de la biología evolutiva. En este marco, el esquema de los modos tecnológicos se convierte en el utensilio más adecuado para tales fines, puesto que en el continuum tecnológico que representa el esquema de Clark, donde cada estadio aparece descargado de referencias histórico-culturales, es fácil interpretar cada nuevo rasgo que se une a la secuencia (los tipos tradicionales) como meras apomorfías biológicas (Foley y Lahr 1997: fig. 3). Foley asume, pues, que los conjuntos líticos constituyen, como los fósiles homínidos, rasgos particulares de la evolución humana y hace suya, por tanto, la máxima una especie, una tecnología. Dejando de lado las críticas que se han vertido sobre esta concepción filética de la producción lítica (Clark 1989) y sobre su observación efectiva en el registro arqueológico (Cosgrove 1999, d'Errico et al. 1998, Bar-Yosef y Kuhn 1999), es necesario reconocer el éxito con que este discurso cladístico ha utilizado el esquema de los modos tecnológicos.

Otro ejemplo significativo de la aplicación de la propuesta de Clark puede verse en diversas contribuciones de Carbonell et al. $(1998,1999)$. En este caso concreto, la terminología se asume como la más adecuada a las investigaciones actuales, que se centran menos en la tipología y cada vez más en la tecnología y las cadenas operativas (Bermúdez de Castro et al. 1999: 192). Semejante opción resulta a primera vista coherente tanto con el momento actual de la investigación, que comentábamos más arriba, como con la trayectoria teórica y metodológica mostrada por este equipo en lo que respecta al estudio de la problemática tecnológica paleolítica (Carbonell et al. 1992) y la necesidad de superar las contradicciones generadas desde el marco historicista (Carbonell et al. 1996: 89).

Después de todo lo observado, las ventajas que, a priori, podríamos enumerar en la aplicación del esquema de los modos tecnológicos son las siguientes:

1. La propia nomenclatura referida a la secuencia tecnológica paleolítica es más aséptica o, si se quiere, más objetiva. La terminología, por tanto, agrupa y describe entidades arqueológicas objetivamente observadas, dejando de lado las referencias clásicas y los posibles remedos histórico-culturales que aún pudieran quedar en ellas. Esta cualidad se ha utilizado de manera significativa en la ya comentada perspectiva cladística o paleoantropológica (usando las mismas estrategias analíticas tanto para el registro biológico como para el cultural).

2. Puede convertirse en un instrumento útil de análisis interregional y global (Schick 1994: 575). Al olvidarse, como decíamos, una estructura basada en los yacimientos epónimos, se evitan las rigideces derivadas de este esquema, que obligaban a comparar de algún modo cada yacimiento con el lugar y las colecciones de referencia que marcaban la pauta. Ahora, por el contrario, se señalan rasgos comunes que agrupan sintéticamente a conjuntos dispares pertenecientes a un mismo ciclo o estadio tecnológico. Desde esta perspectiva, y ante estudios de escala media y grande, se podrá hacer hincapié en los rasgos tecnológicos compartidos por industrias como las de Koobi Fora (Isaac et al. 1997) y las de Olduvai (Leakey 1971), poniendo menos énfasis en las diferencias tipológicas que hicieron etiquetar a las primeras con el nombre de industrias "KBS" o "Karari" cuando se comparaban con el olduvayense normativo.

Otro caso interesante y paradigmático está constituido por la complejidad tipológica observada al final del Paleolítico inferior. Muy a menudo el Paleolítico medio europeo se ha identificado exclusivamente con el musteriense, sin reparar en que éste último constituye un estadio confinado a un tiempo y un espacio dentro de las industrias mesopaleolíticas, que incluirían una multitud más de formas regionales, entre las que se encuentran, por citar algunas, el charentiense francés, el pontiniano italiano, el vasconiense cantábrico o los grupos de Europa oriental, tales como las industrias de Jankovich (Otte 1996: 208). Existen ocasiones en las que resulta conveniente hacer prevalecer los rasgos estructurales que unen frente a los particulares que separan: no solamente ante la problemática de la variabilidad regional del Paleolítico medio europeo, como hemos visto, sino cuando se afrontan temas relacionados con análisis territoriales de diversa índole. 
3. El abandono del esquema de complejos industriales clásicos permite superar los sesgos y contradicciones propias del legado científico. A través del marco actual de conocimiento, que confirma a África como el origen de las radiaciones humanas y de las tecnologías líticas producidas durante el Paleolítico inferior (Aguirre y Carbonell 2001), sería más apropiado utilizar un sistema que asumiera o favoreciera en su nomenclatura este esquema y no otro. Así, el complejo tecnológico que se define principalmente a partir del morfotipo bifaz, que surge en África oriental hace unos 1,5 Ma. (Clark 1994), no acabaría definiéndose a través de los materiales recogidos en las terrazas del valle del Somme francés (Breuil 1939).

\section{ANÁLISIS CRÍTICO DEL MODELO: ¿NOVEDAD O CONTINUIDAD?}

De manera consciente hemos abierto este trabajo abordando sucintamente el marco en el que se sitúa el uso actual del sistema de los modos tecnológicos, las perspectivas que lo han puesto en práctica y las legítimas expectativas que abre. Es momento ahora de acudir al trabajo original y exponer los recursos instrumentales explícitamente expuestos por su autor. De este modo pretendemos establecer una comparación y poner en relieve las marcadas diferencias de fondo existentes entre el esquema original y el marco en el que se está usando en la actualidad.

\section{Un repaso detallado a la propuesta de Clark}

Tal y como se ha comentado, Grahame Clark expone su perspectiva sobre la evolución de las industrias líticas en su libro World Prehistory, publicado por primera vez en 1961. Aquí nos hemos servido de la tercera edición revisada y aumentada de 1977 (23-38), en la que amplía detalladamente sus propuestas. Clark usa como punto de partida para su argumentación el papel preponderante que tuvo la talla lítica en la Prehistoria humana y la variedad y diversidad de técnicas utilizadas a lo largo del tiempo. A partir de aquí, reconoce la existencia de un fondo evolucionista en el comportamiento tecnológico, basado en el perfeccionamiento de los procedimientos a lo largo de amplios periodos de tiempo. Puesto que los objetos líticos favorecen la adaptación al entorno, el proceso evolucionista se ve empujado gracias a la tendencia a producir cada vez tecnologías más eficaces. El concepto de adaptación como motor de la tecnología se usa aquí dentro del contexto de las perspectivas ecológicas de mediados del siglo XX que, posteriormente, darán paso al universo funcionalista (Maschner y Mithen 1996: 5). Estas perspectivas se apoyan en la máxima clásica: la cultura es un medio extrasomático de adaptación (White 1949). El citado proceso de evolución y de incorporación de nuevos elementos técnicos queda definido en 5 estadios diferentes, llamados modos, cuyos rasgos específicos son los siguientes:

Tabla 1. Propuesta de los Modos Tecnológicos de Clark (Clark 1977: tab. 5)

\begin{tabular}{|l|l|}
\hline \multicolumn{1}{|c|}{ Tecnologías líticas dominantes } & \multicolumn{1}{|c|}{ División temporal } \\
\hline Modo 5: componente microlítico en artefactos compuestos & Mesolítico \\
\hline Modo 4: técnica laminar de lascas & Paleolítico superior \\
\hline Modo 3: utensilios sobre lascas obtenidas de núcleos preparados & Paleolítico medio \\
\hline Modo 2: hachas de mano talladas bifacialmente & \} \\
\hline Modo 1: cantos trabajados y lascas & \} Pạleolítico inferior \\
\hline
\end{tabular}


A pesar de que la sucesión desde el Modo 1 al 5 revela una realidad objetiva diacrónicamente, Clark avisaba de varios aspectos que son esenciales a la hora de comprender y aplicar su esquema y que están íntimamente vinculados a su visión ecológica del registro:

- el proceso es continuo y no exhibe diferencias bruscas entre los distintos modos. Es probable que, al entenderse como una adición gradual de rasgos tecnológicos, en un mismo conjunto se combinen técnicas relacionadas con distintos modos.

- la secuencia tecnológica no implica necesariamente una secuencia cronológica, de forma tal que, por ejemplo, el Modo 1 no es obligatoriamente más antiguo que el 2. Dos grupos humanos localizados en el mismo plano temporal se verán empujados a mantener o rechazar elementos técnicamente menos progresivos en función de los condicionantes ambientales.

- el esquema no es universal sincrónicamente y está condicionado por el factor migratorio y la importancia diferencial de la competencia a lo largo del globo: la estasis tecnológica se verá propiciada en regiones periféricas donde las circunstancias ecológicas o demográficas no imponen nuevos retos adaptativos.

Una de las cuestiones más interesantes de la perspectiva de Clark está relacionada con el marco ecológico que, como hemos comentado, es clave en su postura. Sin lugar a dudas, este elemento es notoriamente meritorio y, en muchos aspectos, precursor de ideas utilizadas posteriormente, tales como el rechazo apriorístico a la relación automática e inflexible entre cualquier modo tecnológico y un paréntesis cronológico concreto ${ }^{3}$, la importancia de los condicionantes ambientales en la variabilidad industrial (Mosquera 1995) o el papel de la tecnología como instrumento de adaptación ecológica, visión -esta última- que encontramos perfectamente recogida en la idea de que los artefactos líticos constituyen un recurso para hacer más accesible el medio natural (Carbonell et al. 1983).

Por el contrario, al subrayarse el papel jugado por las migraciones, el movimiento de ideas, la competición y las relaciones centro/periferia en el proceso de innovación tecnológica, parece que el autor aún concede cierta confianza, aunque no hasta el punto que lo hace Foley, a la relación existente entre una tecnología específica y especies biológicas concretas. Esta perspectiva hoy por hoy, precisamente en virtud de los distintos factores de variabilidad que venimos citando (y que Clark alude), resulta poco acertada. La documentación arqueológica y etnográfica se encarga de mostrar que, por ejemplo, la tecnología necesaria para producir láminas no es privativa de Homo sapiens moderno (Bar-Yosef y Kuhn 1999), del mismo modo que los esquemas más elementales, como los propios del Modo 1, no se limitan a los representantes más antiguos del género Homo (Cosgrove 1999).

\section{Contradicciones del modelo}

Sin duda alguna, al margen de estos aspectos, el núcleo por excelencia de la propuesta está relacionado con la propia secuencia de modos y los rasgos seleccionados por el autor para definir e individualizar cada uno de ellos. Para Clark, los rasgos de innovación tecnológica que van apareciendo a lo largo del Paleolítico, y que se van sumando a los conocimientos anteriores, se concretan en determinadas morfologías que constituyen las señas de identidad dominantes y distintivas de cada modo: desde la producción básica de cantos trabajados/núcleos y lascas (que permanecerá como substrato invariable a lo largo de toda la secuencia diacrónica), los grupos humanos van introduciendo en sus repertorios el bifaz (Modo 2), la técnica levallois de predeterminación de núcleos (Modo 3), la talla laminar (Modo 4) y la elaboración de microlitos (Modo 5).

3. Este aspecto está netamente vinculado a discusiones que han florecido posteriormente, tales como la valoración del papel jugado por el factor estilítico (Gamble 1995) o los binomios simple/complejo, eficaz/ineficaz (Torrence 1989: 2) como marcadores útiles con valor cronológico. 
Una observación pausada de este modelo difícilmente halla diferencia alguna entre lo que se propone y las perspectivas ya conocidas de la tradición historicista. Los modos de Clark usan como rasgos diagnósticos

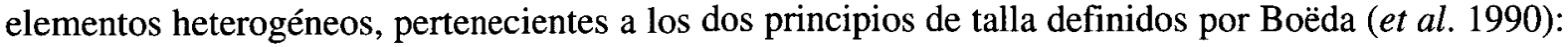
mayoritariamente hacen hincapié en la explotación (producción simple de cantos y lascas, técnica levallois, técnica laminar), pero también acuden a la configuración, al tener en cuenta el bifaz.

Es cierto que, en un vistazo superficial, parece que la propuesta de los modos es netamente tecnológica (al basarse primordialmente en el uso diagnóstico de determinadas técnicas: levallois o laminar), pero el caso del Modo 2 y la excesiva exigüidad con que se determinan los caracteres técnicos invitan a no considerar este modelo exclusivamente como tal. Esta dependencia de un solo objeto o técnica como marcador de un particular estadio, no solo equipara el esquema de Clark con las pautas utilizadas por la tipología convencional, sino que -más importante-automáticamente lo está relacionando con las perspectivas históricoculturales que parecía superar.

Así, en vez de confiar en los procesos o las técnicas que generan artefactos finales para determinar una etapa tecnológica (la impronta distintiva de la tecnología procesual de los últimos años y del modelo de reconstrucción de cadenas operativas), se utiliza un único objeto como referente (situado por definición en la fase final de la cadena productiva). Útil final equivale a tipo en las tipologías tradicionales, las cuales podrían definirse como el estudio y la definición de determinadas categorías descriptivas o formas (Otte 1996: 238), que fundamentalmente tienen en cuenta la panoplia de objetos localizados al final de la secuencia de producción -eso es, los configurados o retocados-, dejando en un segundo plano las etapas productivas de la cadena. Podemos colegir, por tanto, que el Modo 2 se define a partir de un único tipo, que funciona como un fósil-guía tradicional. Su presencia o ausencia es fundamental a la hora de decidir si una industria pertenece o no al Modo 2. Algo parecido ocurre en el resto de los casos, de tal suerte que será la presencia de la talla predeterminada la que defina el carácter Modo 3 de una industria.

Más allá, nos atrevemos a sugerir, que desde el punto de vista metodológico, este modelo constituye un instrumento más limitado y, en algunos casos, menos eficaz que las nomenclaturas anteriores. Si asumimos, como hemos pretendido hacer notar anteriormente, que tanto aquéllas como el esquema de los modos tecnológicos poseen un substrato común basado en el papel de tipos-forma o tipos-técnica, debemos reconocer entonces que, al menos la compleja estructura de facies y grupos regionales, en su afán descriptivo y particularista, hacía más justicia a la variedad y diversidad del registro arqueológico paleolítico. Las perspectivas tradicionales reconocían las diferencias existentes y las posibles relaciones entre tecno-complejos sincrónicos a partir de determinados rasgos tipológicos, de tal suerte que la presencia del bifaz era característica del achelense y la ausencia del bifaz asociado a instrumentos elaborados sobre lascas no preparadas identificaba a otros conjuntos, tales como el tayaciense o el clactoniense. En el sistema de los modos tecnológicos al simplificarse los tipos diagnósticos, se dejan de lado las excepciones a la norma, ahora más rígida que nunca. De este modo, el fósil guía no define ya un particular recuadro de la comentada figura 1 , sino todo un sistema, empobreciendo con ello los instrumentos que tenemos a la hora de establecer definiciones o valoraciones de tipo arqueológico que, paradójicamente, parecían verse reforzadas.

\section{El Modo 2 como eje central de la problemática}

Nos hemos referido ya en varias ocasiones al ejemplo del Modo 2. Ciertamente, éste, tal y como lodefine Clark, supone uno de los problemas fundamentales del sistema, puesto que descansa en un marco epistemológico decimonónico y trasnochado: el uso del bifaz como único referente a la hora de definir, no solamente un tecno-complejo o una facies, sino todo un estadio tecnológico, olvidándose (a pesar del supuesto carácter tecnológico que comentábamos anteriormente) de otros rasgos técnicos asociados (Wenban-Smith 1998: 93). 
Es obvio que detrás del útil bifacial, como objeto final que es, existen unos principios tecnológicos específicos y un particular dominio de la talla (Jones 1994: 262-263) de un orden estructural suficientemente significativo como para rechazar la idea lanzada por algunos investigadores de que la única diferencia, por ejemplo, entre las industrias del Modo 1 y las del Modo 2 se encuentra en la presencia o ausencia de bifaces (Villa 2001: 119). Son esas características, como expondremos más adelante, las que deberían tomarse en cuenta y no simplemente el más significativo de sus resultados. Esta actitud irremediablemente nos conduce a ciertos peligros y callejones sin salida de los que debemos ser conscientes.

El primero de ellos lo constituye el hecho de que no contamos con una respuesta unívoca sobre el significado de los bifaces y su papel en los distintos conjuntos industriales (Gamble 1998). Su presencia en el registro se ha explicado en función de varios factores: económicos y funcionales, asociados al procesamiento de grandes animales (Jones 1980, Díez 1993: 335), tal y como ejemplifican algunos casos (Santonja et al. 1980, Piperno et al. 1999: 86-106); ambientales, en relación a entornos abiertos de pradera, poco arbolados (Butzer 1977, Mithen 1994) o, incluso, sociales (Kohn y Mithen 1999). Desconocemos con total seguridad, por tanto, las claves y múltiples contingencias que determinan la presencia de este objeto en un yacimiento determinado, las cuales pueden estar relacionadas con factores de variabilidad propios del carácter versátil de los objetos durante el Paleolítico inferior y medio (Mosquera 1995: 337).

Si el Modo 2 está constituido exclusivamente por conjuntos donde hay bifaces, ¿dónde quedan situadas otras industrias tradicionalmente paralelas al achelense? ¿deberían ser consideradas Modo 1 ? Este es un problema de cierto alcance, puesto que durante el Pleistoceno medio europeo conocemos la existencia de toda una gama de agrupaciones industriales que carecen del morfotipo bifaz y que, tal y como define Clark, se basan en "cantos/núcleos y lascas" pertenecientes al Modo 1. Entre estas industrias se encuentra el clactoniense, que hace más de dos décadas se interpretó como una industria preparatoria del achelense y, por consiguiente, relacionada con éste (Ohel 1978) y que, en los últimos años, se acepta como una respuesta puntual (sin bifaces) a las variaciones de materia prima que se producen a lo largo del paisaje (Ashton $e t$ al. 1994: 589) o del tiempo (Wenban-Smith 1998: 96). El clactoniense de yacimientos como High Lodge, el tayaciense de Arago -incluso con bifaces (Label 1984) - y el tabauchiense de Bilzinsgleben, Schöningen o Vértesszölös (Mania et al. 1999, Thieme 1999, Dobosi 1990) son industrias que se desarrollan paralelamente al achelense y que se caracterizan fundamentalmente por la configuración de artefactos sobre lascas no preparadas. A pesar de que la perspectiva tradicional aceptaba la existencia de unas industrias de lascas paralelas y diferentes culturalmente al achelense, con origen posiblemente pre-achelense y precursoras del musteriense (Breuil 1932), los estudios recientes apuntan tanto a la similitud tecnológica existente entre ellas (Svoboda 1987) como al hecho de que, salvo la existencia del bifaz, los elementos tecnológicos exhibidos repiten las mismas pautas que las encontrados en el achelense (Villa 2001: 119). Resulta difícil comprender cómo y al amparo de qué similitudes tecnológicas, en el marco propuesto por Clark, estas industrias que no cuentan con los tipos requeridos (el bifaz o la técnica levallois), deberían entrar en el Modo 1. A pesar de que, por otro lado, parecen vincularse con el Modo 2, la escasa flexibilidad del esquema "tipológico" de Clark las aleja irremediablemente de la norma.

\section{HACIA LA REDEFINICIÓN DEL SISTEMA DE MODOS TECNOLÓGICOS}

Más de treinta años después de que la propuesta de Clark viera la luz, existe una evidente falta de acuerdo en lo que respecta a su puesta en práctica. Los investigadores han hecho suyo el uso de la nomenclatura, haciendo que ésta sirva a intereses y sensibilidades distintas, sin que hasta el momento se hayan producido revisiones criticas del esquema, adaptaciones o declaraciones explícitas que sometan a una comparación con la propuesta original aquello a lo que nos referimos cuando hablamos de un Modo tecnológico concreto. 
Es cierto que algunos investigadores han expuesto con cierto detalle su perspectiva particular sobre este modelo, asociada a una visión tecnológica y procesual más ambiciosa que la observada en el marco de la propuesta de Clark, aunque ésta se ha expresado en el restringido marco de un yacimiento específico (Carbonell et al. 1998), lo cual limita en gran medida las caracterizaciones a nivel global de un modelo cuyo poder metodológico, paradójicamente, parece basarse en los estudios a nivel regional y global.

Entre los autores que hacen uso de esta nomenclatura, podemos establecer una neta distinción entre aquellos que la aplican siguiendo estrictamente la visión de Clark (fundamentalmente, y de manera bastante generalizada, en el entorno de la arqueología anglosajona -Schick y Toth 2001: 55, 70-) y aquellos otros que han dotado al sistema de unos recursos más elaborados y acordes con lo que serían las expectativas presentadas anteriormente (Carbonell et al. 1998). Seguramente ambos comparten el reconocimiento de la misma cualidad básica del modelo (su neutralidad cultural), pero divergen sustancialmente en su explicitación.

Desde nuestro punto de vista creemos que si lo que se desea es realmente superar el marco tradicional del historicismo cultural y hacer viables aquellas ventajas que citábamos con cierto detalle en el apartado 2 , el modelo original de Clark es insuficiente y necesita ser redefinido en términos globales. Para ello, deberían tenerse en cuenta criterios tecnológicos unificados y homogéneos que se aplicasen a todos los estadios, sin distinción (que también deberían servir, en contra de lo que hemos visto, para el caso del Modo 2).

\section{Los Modos y tecnología en el Paleolítico inferior y medio}

Pretendemos, en el sentido que acabamos de comentar en el anterior párrafo, apuntar y someter a cierta discusión determinados datos tecnológicos que pueden servir a la hora de definir con detalle los distintos rasgos implicados en los modos del Paleolítico inferior y medio.

- Modo 1: el predominio de la producción. Este primer estadio es reconocido hace 2,5 Ma. en África oriental (Semaw 2000) y ha sido identificado como una tecnología del mínimo esfuerzo (Isaac et al. 1997: 296), cuyas características se explican detalladamente en varios trabajos (Toth 1985, Ludwig y Harris 1998). El objetivo fundamental del Modo 1 es la simple producción masiva y sistemática de lascas con filos naturales cortantes (Toth 1985: 118). Si asumimos este aspecto esencial y utilizamos el concepto de los grandes principios de talla expuestos por Boëda (et al. 1990: 43), lo definiríamos por la puesta en práctica de cadenas operativas destinadas a la producción. A pesar de que, en este periodo, los esquemas de gestión son ya casi tan variados como los que encontraremos más adelante (unipolar, bipolar, multipolar y centrípeto), éstos constituyen simples subproductos del proceso de obtención de lascas, en los que las formas de los núcleos a menudo se encuentran condicionadas por la morfología original de los cantos matrices (Toth 1985: fig. 5). De acuerdo con la estrategia fundamental que estamos comentando, la existencia de procesos de configuración en instrumentos de pequeño formato es muy limitada y se caracteriza por el retoque marginal, no estructurado y con tendencia a la delineación no sistemática de los filos que se transforman.

- Modo 2: la configuración y estandarización de las formas. Si hacemos del achelense el paradigma del Modo 2 y, por tanto, asumimos la idea de que el bifaz constituye el ejemplo más significativo de esta innovación tecnológica en el registro arqueológico, deberíamos comenzar por poner de manifiesto los rasgos tecnológicos que constituyen el substrato de tal comportamiento, de tal manera que estos sea identificados como rasgos de referencia. Algunos autores han negado la existencia de una intención o habilidad técnica específica en la producción del instrumental bifacial, puesto que -según ellos- éste no constituiría otra cosa que el resultado final, en cierto modo involuntario, de la producción de lascas a partir de una matriz 
cualquiera (Noble y Davidson 1996: 196-198). Si, por el contrario, aceptamos que estos objetos suponen el resultado, mediante unos requerimientos tecnológicos concretos, de una estructura mental previa, entonces la perspectiva es distinta.

La producción de objetos de gran formato (hendedores y bifaces) requiere, según algunos autores, la puesta en marcha de los principios técnicos de estandarización y predeterminación (Texier y Roche 1995). El primero, implica la configuración sistemática de formas concretas a través de la adopción de gestos técnicos recurrentes, que implican la delineación de filos mediante la talla y el retoque, ajustándolos a las características del soporte (Carbonell et al. 1999: 321-323; Ambrose 2001: 1750). Uno de los elementos técnicos que caracteriza al achelense es la producción de grandes lascas, posteriormente transformadas en utensilios bifaciales. La puesta en marcha de esta técnica puede implicar (Texier y Roche 1995: 408, Carbonell et al. 1999: 312) la preparación previa y cuidadosa del núcleo para la obtención de la gran lasca, lo que implica su predeterminación.

Los rasgos tecnológicos más evidentes de este proceso son, por tanto, la configuración de formas (que se produce estandarizada o repetidamente) y, en ocasiones, la predeterminación del soporte que se va a transformar. Estos aspectos, fundamentalmente el primero, aluden al segundo principio de talla definido por Boëda et al. (1990), el de la configuración. Asumimos que tal aspecto, si realmente representa un fenómeno tecnológico definido, podrá extenderse a todo el repertorio producido y ser observado tanto en la elaboración sistemática (estandarizada) de los tradicionales objetos de gran formato (bifaces y hendedores), como en objetos de pequeño formato (filos configurados mediante el retoque que delinean formas variadas pero significativamente repetidas: raederas, perforadores, raspadores...). Reconoceríamos, por tanto, que el Modo 2 tiene un elemento de pequeño formato, no bifacial, que puede ir acompañando a éste, pero en ocasiones manifestares al margen (Wenban-Smith 1998: 93). El concepto Modo 2, apoyado por tanto en la configuración, daría cabida a aquellas industrias que consisten en el nada explícito binomio propuesto por Clark de cantos y lascas, pero que, más allá de la simple producción ad hoc de filos cortantes, ponen en práctica la configuración repetida de formas mediante el retoque (aplicando el mismo esquema básico que define a un bifaz).

- Modo 3 y el continuo tecnológico. La definición del Modo 3 se enmarca en una reconocida problemática que se basa en lo difícil que resulta establecer una neta diferenciación o distinción entre el Paleolítico inferior y el medio. Obviamente, este tránsito ha sido definido como un continuum tecnológico en el que los rasgos de distinción son difíciles de aislar (Clark 1988: 236-237). Algunos autores han llegado más allá y han propuesto que el Paleolítico medio no tiene significado como entidad tecnológica específica (Boëda 1991: 37), sino que más bien representa simplemente un estadio particular del desarrollo de las tecnologías anteriores (Rolland 1999: 316). Estos aspectos se verían reforzados si tenemos en cuenta el solapamiento cronológico entre las industrias del Modo 2 y las del 3 en el Pleistoceno medio (Rolland 1999: fig. 3). Hablaríamos, así, de una diferenciación de "grado" más que estructural (Carbonell et al. 1998: 395, 417), cuya unificación en lo que llamamos Paleolítico medio respondería exclusivamente -para algunos-a criterios de conveniencia (Gamble 1990: 134).

Este debate es crucial, a nuestro modo de ver, en el proceso de distinción del Modo 3 como estadio independiente (dado que aquí estamos teniendo en cuenta criterios tecnológicos y no formales). Entre la variedad de factores que han sido señalados para definir al Modo 3 (Rolland 1999: tab. 1), los principales, en torno a los cuales gira el resto, son dos: en primer lugar, tal y como cita Clark, la gestión predeterminada de núcleos tipo levallois para la obtención de lascas y puntas; después, el desarrollo de los procesos de configuración a través de un perfeccionamiento del retoque (tipo Quina, bifacial, plano) y, por consiguiente, el aumento y diversificación de la tipología de artefactos configurados de pequeño formato. Ambos aspectos tecnológicos constituyen exactamente los mismos que hemos utilizado para definir al Modo 2: configuración/estandarización 
de formas (de gran y pequeño formato) y predeterminación (fundamentalmente observada en la producción de grandes lascas posteriormente utilizadas en la configuración de bifaces y hendedores).

Algunos investigadores han confiado en criterios puramente convencionales para distinguir al Paleolítico inferior del medio, tales como la frontera cronológica Pleistoceno inferior y medio (Santonja y Pérez-González 1997). Quizás esta opción pueda sernos de utilidad en determinados momentos, aunque el comentado solapamiento cronológico que ya hemos citado dificulta su posible valor instrumental. Otro factor a tener en cuenta; que oscurece una perspectiva meramente tecnológica, está constituido por el hecho de que la gestión levallois es un instrumento diagnóstico conflictivo. Así, se produce la paradoja de que está presente en determinados conjuntos achelenses, mientras que muchas industrias del Paleolítico medio no se caracterizan por este rasgo (McBrearty y Brooks 2000: 485).

Tras todo lo dicho, podemos concluir que la distinción entre ambos puntos del segmento se basa exclusivamente, como decíamos, en aspectos de grado, pero no de fondo: dimensionales, con la tendencia a la predeterminación y configuración de objetos de pequeño formato; y porcentuales, con la tendencia a la diversificación de formas. El problema, y es aquí donde el concepto continuum cobra mayor sentido, es saber si contamos con los recursos metodológicos necesarios para definir e identificar el significado de dicha tendencia. Si la respuesta es no (y, a nuestro juicio, ese es el lugar en el que nos encontramos), la distinción entre los Modos 2 y 3 perdería fuerza y su aceptación se vería enormemente debilita. Esto es así porque, si bien el simple método comparativo nos permite reconocer la variación tecnológica de carácter diacrónico en una misma secuencia arqueológica (para la que nos servirían tanto el esquema de los modos tecnológicos, como otro más tradicional), la situación se complica enormemente al acotar dichas tendencias entre conjuntos diferentes. En este caso, el uso de la nomenclatura Modo 2-Modo 3, cuya principal ventaja residía en las relaciones a gran escala, se haría menos consistente.

\section{DISCUSIÓN}

A lo largo de estas páginas hemos sometido a revisión algunos aspectos relacionados con la propuesta metodológica de Grahame Clark sobre los modos tecnológicos y su validez en el marco de los estudios paleolíticos. Nos hemos centrado en la problemática del Paleolítico inferior y medio, porque estamos convencidos de que es aquí donde se encuentran las mayores dificultades del esquema, principalmente en lo que concierne a la definición clásica del Modo 2 y la validez de criterios para el reconocimiento diferenciado del Modo 3. En todo caso, la complejidad técnica que supone la incorporación de estadios tecnológicos posteriores (McBrearty y Brooks 2000; Ambrose 2001) constituye un apartado que necesitaría una atención más detallada.

El esquema de los Modos tecnológicos propuesto por Clark en los años 60 supone, para la mayor parte de investigadores que lo usan, un magnífico instrumento a la hora de aportar una nomenclatura neutra y aséptica, no historicista, ajena a un excesivo determinismo cultural, no eurocéntrica y cientifista. (Schick y Toth 2001). Este, seguramente, puede ser un punto de encuentro básico entre todos los investigadores que, en un lugar u otro, lo usan. Sin embargo, como hemos visto en el primer apartado, existe una evidente falta de unanimidad, y por consiguiente una clara confusión, en torno a lo que cada una de las etapas encierra. En algunos casos, fundamentalmente en el entorno anglosajón, los conceptos se han mantenido más fieles a la idea original de Clark, mientras que en otros sitios se han pretendido dotar de unos instrumentos novedosos, más acordes con los intereses teóricos perseguidos.

Intentando superar este problema de discordancia, el punto de vista que hemos defendido en estas páginas pasa indudablemente por tener en cuenta los referentes de la tradición historicista europea como telón de fondo y por el deseo de superar sus limitaciones (aceptando el valor evidente de sus logros), en la misma 
línea que lo pueden haber intentado las distintas perspectivas procesuales de análisis lítico que se están imponiendo a las tipologías clásicas. Fuera de este objetivo esencial, el uso de una taxonomía u otra (la clásica o la de Clark) en la definición de los cambios tecnológicos carecerá de verdadera importancia y se apoyará simplemente en el uso de un lenguaje más o menos en boga. En este sentido, hemos pretendido razonar el aserto de que el sistema de los Modos tecnológicos en su versión original, aparte de haber aportado un lenguaje impactante y de una fuerza innegable, no responde a las expectativas de fondo señaladas: aquellas que pretenderían resaltar los rasgos tecnológicos comunes frente a las diferencias tipológicas particulares, con objeto de establecer un instrumento de trabajo que permitiera interrelacionar fenómenos arqueológicos distintos o distantes geográficamente, para la generación de síntesis regionales o globales.

La muestra más evidente de estas limitaciones está constituida, sin duda, por la problemática del Modo 2 y el uso de un tipo único (aunque sea un tipo con una gran fuerza, como el bifaz) como parámetro de referencia absoluto. Este hecho sitúa de nuevo el modelo diagnóstico en el punto de partida que se pretende superar: presencia/ausencia de determinado objeto final. El hecho de que el sistema de los Modos tecnológicos no ha funcionado como el supuesto marco novedoso y aventajado que se suponía descansa en un dato teórico muy significativo. Muy a menudo hemos asumido la siguiente equivalencia (Bermúdez de Castro et al. 1999: 193, Diez Martín 1999; Rodríguez 2001): Modo 1= olduvayense; Modo 2= achelense; Modo 3= musteriense. A nuestro juicio, la activación de tal estructura, no solamente no está aportando un marco nuevo, sino que repite el anterior y, además, limita severamente el espectro taxonómico clásico. Como hemos advertido, se convierte en un instrumento incluso menos útil que el tradicional. Es difícil comprender, como hemos intentado mostrar a lo largo de estas páginas, en qué medida los modos tecnológicos se llenan de contenidos nuevos cuando acuden al marco clásico de referencia (con sus sesgos interpretativos). Permítasenos usar una expresión coloquial que glosa perfectamente esta situación: "para tal viaje no necesitábamos estas alforjas". El hecho de que la nomenclatura de los modos se convierta en un decorado de cartón piedra no es suficiente para que su poder teórico y metodológico alcance un valor práctico consistente.

Hemos intentado poner de manifiesto la necesidad de redefinir el significado de los modos y dotarlos de un contenido tecnológico que pueda servir para superar y aventajar las taxonomías clásicas como instrumento de trabajo que refuerce la utilidad del sistema para la resolución de problemas arqueológicos de gran escala. El binomio producción/configuración y toda la carga operativa y tecnológica que lo apoya puede servirnos como punto de partida para la definición de los dos primeros estadios de la elaboración tecnológica (Modos 1 y 2). Es necesario, como hemos visto, profundizar en la siguiente etapa (Modo 3) y su diferenciación objetiva respecto a la anterior. Para ello es preciso tener en cuenta la medida en la que la predeterminación del instrumental de pequeño y gran formato responde a conceptos netamente diferentes. La idea de predeterminación es, sin duda y en todo caso, el mejor argumento que podemos utilizar en la caracterización del Modo 3 como estadio consistente o como mero concepto de grado.

Finalmente, reconociendo la importancia del modelo de Clark como elemento generador de nuevas ideas, hemos de hacer hincapié en que tal esquema debe perfeccionarse, haciendo posible una mayor adecuación entre los instrumentos de trabajo con que contamos y el objetivo de establecer un acercamiento más completo a las realidad paleolítica a través de su producción tecnológica.

\section{AGRADECIMIENTOS}

El presente trabajo ha sido posible gracias a la concesión de una beca postdoctoral de investigación por el Gobierno Vasco en la Universidad de Indiana (EE.UU.), entre los años 2000 y 2002. 


\section{BIBLIOGRAFÍA}

ADAMS, W. (1998): The philosophical roots of anthropology. CSLI Publications. Standford.

AGUIRRE, E. y CARBONELL, E. (2001): "Early human expansion into Eurasia: The Atapuerca evidence", Quaternary International 75: 11-18.

AMBROSE, S. (2001): "Palaeolithic technology and human evolution", Science 291: 1748-1753.

BAR-YOSEF, O. y KUHN, S. (1999): "The big deal about blades: laminar technology and human evolution", American Anthropologist 101: 322-338.

BERMÚDEZ DE CASTRO, J. M.; ARSUAGA, J. L.; CARBONELL, E. y RODRÍGUEZ, J., eds. (1999): Atapuerca, nuestros antecesores. Junta de Castilla y León.Salamanca

BISHOP, W. y CLARK, J. (1967): Background to evolution in Africa. Chicago University Press. Chicago

BOËDA, E. (1991): “Approche de la variabilité des systèmes de production lithique des industries du Paléolithique inférieur et moyen. Chronique d'une variabilité attendue", Techniques et culture 17-18:37-39.

BOËDA, E.; GENESTE, J. M. y MEIGNEN, L. (1990): "Identification de chaînes opératoires lithiques du Paléolithique ancien et moyen", Paléo 2: 43-77.

BREUIL, H. (1936): "Les industries à éclat du Paléolithique ancien", Préhistorie I (2): 16-190.

- (1939): "The Pleistocene succession in the Somme Valley", P.P.S. 5: 33.

BUTZER, K. (1977): "Environment, culture and human evolution", American Scientist 65: 572-584.

CARBONELL, E.; MOSQUERA, M.; OLLÉ, A.; RODRÍGUEZ, X. P.; SALA, R.; VAQUERO, M. y VERGÈS, J. M. (1992): New elements of the logical analytic system. Cahier Noir, 6. Tarragona

CARBONELL, E.; SALA, R. y CABAÑAS, A. (1996): "Typology and technology of the Acheulean non-flint assemblages in the North-eastern Iberian Peninsula", en N. Moloney, L. Raposo y M. Santonja (eds.), Non-flint stone tools and the Palaeolithic occupation of the Iberian Peninsula: 89-95. BAR, International Series. Oxford.

CARBONELL, E.; RODRÍGUEZ, X. P. y SALA, R. (1998): "Secuencia diacrónica de sistemas litotécnicos en la Sierra de Atapuerca (Burgos)”, en E. Aguirre (ed.), Atapuerca y la evolución humana: 393-419. Fundación Ramón Areces. Madrid.

CARBONELL, E.; MÁRQUEZ, B.; MOSQUERA, M.; OLLÉ, A.; RODRÍGUEZ, X. P.; SALA, R. y VERGÈS, J. M. (1999): "El Modo 2 en Galería. Análisis de la industria lítica y sus procesos técnicos", en E. Carbonell, A. Rosas y J.C. Díez (eds.), Atapuerca: ocupaciones humanas y paleoecología del yacimiento de Galería: 299-352. Junta de Castilla y León. Valladolid.

COSGROVE, R. (1999). "Forty-two degrees south: the archaeology of Late Pleistocene Tasmania Palaeoecology and Pleistocene occupation in south central Tasmania", Journal of World Prehistory 13: $357-402$.

CHILDE, G. (1929): The Danube in Prehistory. Oxford University Press. Oxford.

CLARK, J. D. (1988): “The Middle Stone Age of East Africa and the beginnings of regional identity", Journal of World Prehistory 2: 235-305.

- (1994): "The acheulean industrial complex in Africa and elsewhere", en R. Corruccini y R. Ciochon (eds.), Integrative paths to the past: Paleoanthropological advances in honor of F. Clark Howell: 451-469. Prentice Hall. Nueva York.

CLARK, G. (1977): World Prehistory in new perspective (tercera edición). Cambridge University Press. Cambridge.

(1989): "Alternative models of Pleistocene biocultural evolution: a response to Foley", Antiquity 63: 153-162.

COLLINS, D. (1969): “Culture traditions and environment of Early Man”, Current Anthropology 10: 267-316. 
DÍEZ FERNÁNDEZ-LOMANA, C. (1993): Zooarqueología de Atapuerca (Burgos) e implicaciones paleoeconómicas del estudio tafonómico de yacimientos del Pleistoceno medio. Universidad Complutense. Madrid.

DÍEZ MARTÍN, F. (1999): "Sobre el cambio tecnológico: el paradigma gradualista y la transición entre el Paleolítico inferior y el medio", Zephyrus LII: 53-78.

DOBOSI, V. (1990): "Description of the archaeological material", en M. Kretzoi y V. Dobosi (eds.), Vértesszölös. Man, site and culture: 311-395. Akadémiai Kiadó. Budapest.

d'ERRICO, F.;ZILHAO, J.; JULIEN, M.; BAFFIER, D. y PELEGRIN, D. (1998): "Neanderthal acculturation in Western Europe?", Current Antrhopology 39: 1-44.

FOLEY, R. (1987): "Hominid species and stone-tool assemblages: how are they related?", Antiquity 61: 380-392.

FOLEY, R. y LAHR, M. (1997): "Mode 3 technologies and the evolution of modern humans", Cambridge Journal of Archaeology 7: 3-36.

GAMBLE, C. (1990): El poblamiento paleolítico de Europa. Crítica. Barcelona.

- (1995): "Interpretation in the Palaeolithic", en I. Hodder, M. Shanks, A. Alexandri, V. Buchli y L. Carman (eds.), Interpreting archaeology. Finding meaning in the past: 87-91. Rotuledge. Londres

_ (1998): "Handaxes and Palaeolithic individuals", en N. Ashton, F. Healy and P. Pettitt (eds.), Stone Age archaeology. Essays in honour of John Wymer: 105-109. Oxbow. Oxford.

GOWLETT, J. (1996): "The frameworks of early hominid social systems", en J. Steele y S. Shennan (eds.), The archaeology of human ancestry. Power, sex and tradition: 135-183. Routledge. Londres.

ISAAC, G.; HARRIS, J. y KROLL, E. (1997): "The stone artefact assemblages: a comparative study", en G. Isaac y B. Isaac (eds), Koobi Fora research project 5: 262-299. Clarendon Press. Oxford.

JONES, P. (1980): "Experimental butchery with modern stone tools and its relevance for Palaeolithic archaeology", World Prehistory 12: 153-163.

JONES, P. (1994): "Results of experimental work in relation to the stone industries of Olduvai Gorge", en M.D. Leakey y D.A. Roe (eds), Olduvai Gorge 5: 254-298. Cambridge University Press. Cambridge.

KOHN, M. y MITHEN, S. (1999): "Handaxes: products of sexual selection?", Antiquity 73: 518-526.

KUHN, S. y SARTHER, C. (2000): "Food, lies and paleoanthropology. Social theory and the evolution of sharing in humans", en M. Schiffer (ed.), Social theory in archaeology: 79-96. University of Utah Press. Salt Lake City.

LEAKEY, M. (1971): Olduvai Gorge: Excavations in Bed I and II, 1960-1963. Cambridge University Press. Cambridge.

LEBEL, S. (1984): La Caune de l'Arago. Étude des assemblages lithiques d'une grotte du Pléistocène moyen en France. Tesis doctoral inédita, Université Paris VI.

LUDWIG, B. y HARRIS, J. (1998): “Towards a technological reassesment of East African Plio-Pleistocene lithic assemblages”, en M. Petraglia y R. Korisettar (eds.), Early human behaviour in global context: 84-107. Routledge. Londres.

MANIA, D.; MANIA, U. y VLECK, E. (1999): "The Bilzingsleben site. Homo erectus, his culture and his ecosphere", en H. Ullrich (ed.), Hominid evolution. Lifestyles and survival strategies: 283-314. Archaea. Berlín.

MASCHNER, H. y MITHEN, S. (1996): "Darwinian archaeology. An introductory essay”, en H. Maschner (ed.), Darwinian Archaeology: 3-14. Plenum Press. Nueva York.

McBREARTY, S. y BROOKS, S. (2000): "The revolution that wasn't: a new interpretation of the origin of modern human behavior", Journal of Human Evolution 39: 453-563.

MITHEN, S. (1994): "Technology and society during the Middle Pleistocene", Cambridge Archaeological Journal 4: 3-32. 
MOSQUERA, M. (1995): Procesos técnicos y variabilidad de la industria lítica del Pleistoceno medio de la Meseta: Sierra de Atapuerca, Torralba, Ambrona y Áridos. Tesis doctoral inédita, Universidad Complutense de Madrid.

NOBLE, W. y DAVIDSON, I. (1996): Human evolution, language and mind. Cambridge University Press. Cambridge.

OHEL, M. (1979): “The Clactonian: an independent complex or an integral part of the Acheulean?", Current Anthropology 20: 685-713.

OTTE, M. (1996): Le paléolithique inférieur et moyen en Europe. Armand Colin. París.

PANERA, J. y RUBIO, S. (1999): "Estudio tecnomorfológico de la industria lítica de Ambrona", Trabajos de Prehistoria 54: 58-71.

PIPERNO, M.; CASSOLI, F.; TAGLIACOZZO, A. y FIORE, I. (1999): "I livelli della serie di Notarchirico", en M. Piperno (ed.), Notarchirico. Un sito del Pleistocene medio iniziale nel bacino di Venosa: 75-135. Ossana. Venosa.

RENFREW, C. y BAHN, P. (1991): Archaeology. Theories, methods and practice. Thames and Hudson. Nueva York.

RODRÍGUEZ, X.P. (2001): “Evolució tècnica dels homínids", L'Avenç 259: 35-39.

ROLLAND, N. (1999): "The Middle Palaeolithic as development stage: evidence form technology, subsistence, settlement systems and hominid socio-ecology", en H. Ullrich (ed.), Hominid evolution. Lifestyles and survival strategies: $315-334$. Archaea. Berlin.

SANTONJA, M. (1996): "The Lower Palaeolithic of Spain: sites, raw material and occupation of the land", en N. Moloney, L. Raposo y M. Santonja (eds.), Non-flint stone tools and the Palaeolithic occupation of the Iberian Peninsula: 1-20. BAR International Series 649. Oxford.

SANTONJA, M. y PÉREZ-GONZÁLEZ, A. (1997): "Los yacimientos achelenses en terrazas fluviales de la Meseta central española", Cuaternario Ibérico 9: 224-234.

SANTONJA, M.; LÓPEZ MARTÍNEZ, N. y PÉREZ-GONZÁLEZ, A. (1980): Ocupaciones achelenses en el Valle del Jarama (Arganda, Madrid). Diputación de Madrid. Madrid.

SCHICK, K. (1994): "The Movius line reconsidered. Perspectives on the Earlier Paleolithic of Eastern Asia", en R. Corruccini y R. Ciocon (eds.), Integrative paths to the past: 569-596. Prentice Hall. New Jersey.

SCHICK, K. y TOTH, N. (2001): "Paleoanthropology at the Millennium", en G. Feinman y D. Price (eds.), Archaeology at the Millenium: 39-108. Plenum. Nueva York.

SEMAW, S. (2000): "The world's oldest stone artefacts from Gona, Ethiopia: their implications for understanding stone technology and patterns of human evolution between 2,6-1,5 million years ago", Journal of Archaeological Science 27, 1197-1214.

SHANKS, M. y TILLEY, C. (1987): Social theory and archaeology. Polity Press. Oxford.

SVOBODA, J. (1987): "Lithic industries of the Arago, Vértesszöllös and Bilzingsleben hominids: comparison and evolutionary interpretation", Current Anthropology 28: 219-227.

TEXIER, P-J. y ROCHE, H. (1995): "The impact of predetermination on the development of some acheulean chaînes opératoires", en J.M. Bermúdez de Castro, J.L. Arsuaga y E. Carbonell (eds.), Evolución humana en Europa y los yacimientos de la Sierra de Atapuerca: 403-420. Junta de Castilla y León. Valladolid.

THIEME, H. (1999): “Lower Palaeolithic throwing spears and other wooden implements from Schöningen, Germany", en H. Ullrich (ed.), Hominid evolution. Lifestyles and survival strategies: 383-395. Archaea. Berlín.

TIEU, L. T. (1991): Palaeolithic pebble industries in Europe. Akadémiai Kiadó. Budapest.

TORRENCE, R. (1989): “Tools as optimal solutions”, en R. Torrence (ed.), Time, energy and stone tools: 1-16. Cambridge Unviersity Press. Cambridge. 
TOTH, N. (1982): The stone technologies of Early Hominids at Koobi Fora, Kenya: an experimental approach. Tesis doctoral inédita. Universidad de California en Berkeley.

(1985): "The Oldowan reassessed: a close look at Early Stone artifacts", Journal of Achaeological Science 12: 101-120.

VILLA, P. (2001): "Early Italy and the colonization of Western Europe", Quaternary International 75: 113-130.

WENBAN-SMITH, F. (1998): "Clactonian and Acheulian industries in Britain: their chronology and significance reconsidered”, en N. Ashton, F. Healy y P. Pettitt (eds.), Stone Age archaeology. Essays in honour of John Wymer: 90-97. Oxbow. Oxford.

WHITE, L. (1949): The science of culture. Grove Press. Nueva York. 